\section{A Patient with Upper Cervical Spinal Cord Infarction Presenting with the Sudden Onset of Severe Occipital Headache Followed by Occipital Neuralgia}

\section{Abstract}

A 78-year-old man suddenly developed a severe occipital headache, followed by right greater occipital neuralgia (ON). Brain MR imaging revealed a spinal cord infarction in the territory of the right posterior spinal artery (PSA) at the C2 level without vertebral artery (VA) dissection. Even without VA dissection, upper cervical PSA syndrome could cause severe occipital headache. Furthermore, ipsilateral ON could follow it. The present case suggests that severe occipital headache and ON can occur in a patient with upper cervical PSA syndrome without VA dissection.

Keywords: Spinal cord infarction; Posterior spinal artery; Headache; Occipital neuralgia

Received: January 28, 2019; Accepted: July 22, 2019; Published: July 30, 2019

\author{
Hisashi Ito ${ }^{1}$, Ai Seki², \\ Shigeru Fukutake', \\ Sanae Odake ${ }^{1,3}$, \\ Terunori Sano ${ }^{4}$, \\ Yuji Uchida², \\ Hiroshi Kitahara ${ }^{5}$ and \\ Tetsumasa Kamei ${ }^{1}$
}

1 Department of Neurology, Shonan Fujisawa Tokushukai Hospital, Fujisawa, Japan

2 Department of Emergency Medicine, Shonan Fujisawa Tokushukai Hospital, Fujisawa, Japan

3 Department of Internal Medicine, Sodegaura Satsuki-dai Hospital, Sodegaura, Japan

4 Department of Pathology and Laboratory Medicine, National Center Hospital, National Center of Neurology and Psychiatry, Kodaira, Japan

5 Emergency Care Center, Yokosuka General Hospital Uwamachi, Yokosuka, Japan

*Corresponding author: Dr. Hisashi Ito

\section{Introduction}

Upper cervical spinal cord infarction in the region supplied by the posterior spinal artery (PSA) is extremely rare. One of the causes of spinal cord infarction is vertebral artery (VA) dissection, which occurs headache or neck pain at a high rate [1-9]. We herein report a patient with upper cervical PSA syndrome without VA dissection. He suddenly developed the worst occipital headache of his life, followed by ipsilateral occipital neuralgia (ON).

\section{Case Report}

A 78-year-old man with hypertension, diabetes mellitus, and dyslipidemia, suddenly developed the most severe throbbing pain that he had ever experienced in the right occipital region with vomiting. An abnormal sensation of the right upper limb occurred simultaneously. He was transported to our hospital by ambulance.

On examination, he was initially hypertensive, at $174 / 86 \mathrm{mmHg}$. He presented with neither cranial nerve abnormality nor muscle weakness; however, we observed severe disturbance of deep sensation in the right upper limb with sensory ataxia. Tenderness was observed in the upper cervical vertebra despite being negative for meningeal signs. Hyperglycemia (289 mg/dL) with high HbA1c levels (9.3\%, NGSP) and hyperlipidemia (TG $175 \mathrm{mg} /$ $\mathrm{dL}$, LDL $158 \mathrm{mg} / \mathrm{dL}$ ) were observed. Cerebrospinal fluid revealed neither erythrocytes nor pleocytosis but showed high protein

\section{”hisashi.ito@tokushukai.jp}

Department of Neurology, Shonan Fujisawa Tokushukai Hospital, 251-0041, Fujisawa, Japan.

Tel: $+81-466-35-1177$

Fax: +81-466-35-1300

Citation: Ito H, Seki A, Fukutake S, Odake S, Sano T, et al. (2019) A Patient with Upper Cervical Spinal Cord Infarction Presenting with the Sudden Onset of Severe Occipital Headache Followed by Occipital Neuralgia. J Neurol Neurosci Vol.10 No.4:300

levels $\left(71.2 \mathrm{mg} / \mathrm{dL}\right.$ ). The opening pressure was $200 \mathrm{~mm} \mathrm{H}_{2} \mathrm{O}$. T2weighted and FLAIR MR imaging of the brain demonstrated a high-intensity area in a right dorsal lesion of the cervical cord at the C2 level [10], which was supplied by the right PSA (Figure 1). 3D-CT angiography revealed severe stenosis of the right vertebral artery (VA) without a string sign or double lumen. No aneurysm 

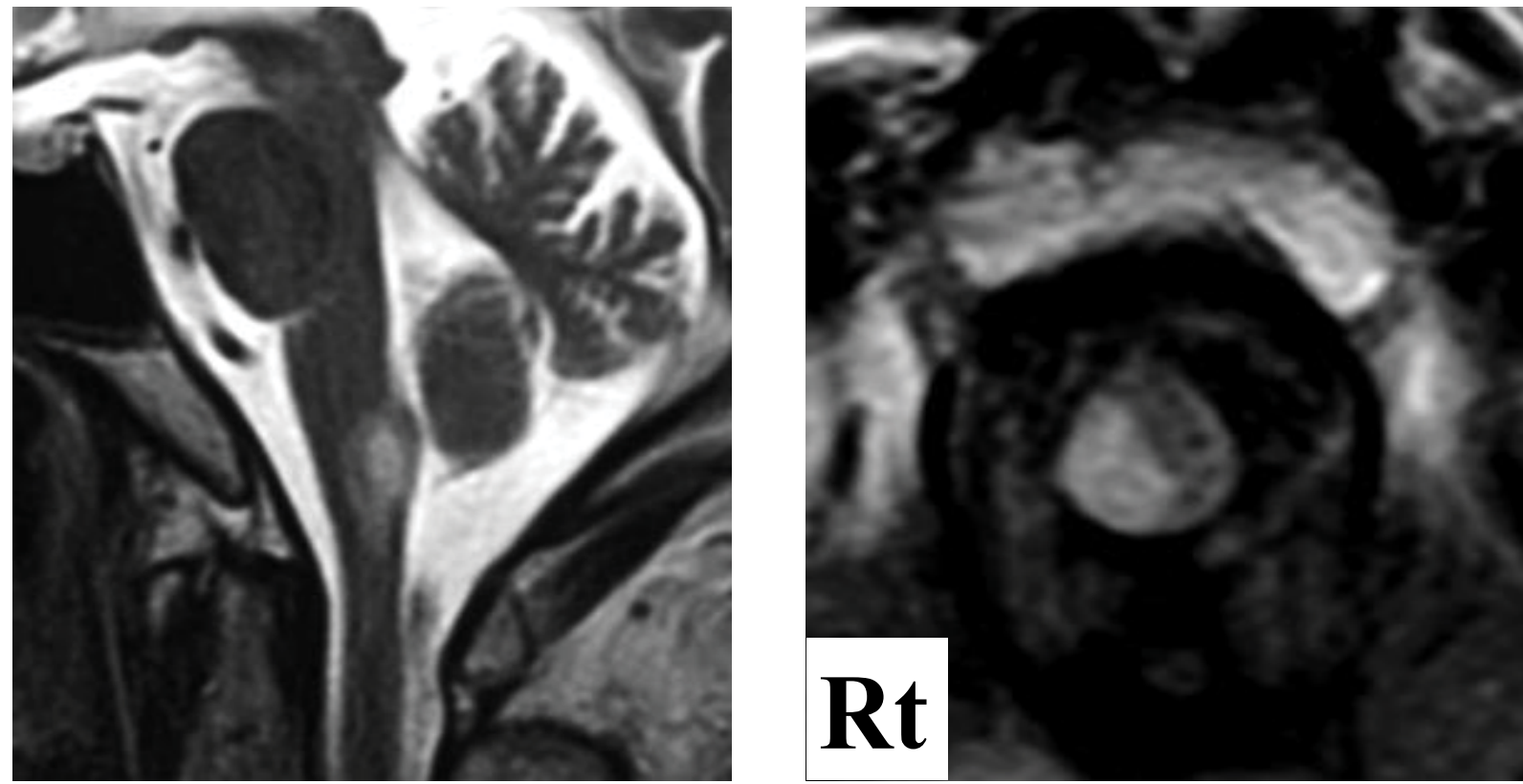

Figure 1 T2-weighted (sagittal view) and FLAIR (axial view) MR imaging. High intensity lesion corresponds to the C2 level [10].

was detected (Figure 2). The right-sided N20 was poorly evoked among somatosensory evoked potentials.

The severe occipital headache was improved with $400 \mathrm{mg}$ of acetaminophen; however, a sharp and jabbing pain in the right greater occipital nerve region developed, followed by hypesthesia and hypalgesia, which was consistent with greater ON. A dose of $100 \mathrm{mg}$ of carbamazepine daily showed good efficacy against ON. Sensory disturbance improved gradually, and he was discharged on the 16th day after admission.

\section{Discussion}

Spinal cord infarction, especially in the territory of the PSA, is exceedingly rare. PSA syndrome due to spinal cord infarction is characterized by acute or sudden onset of pain, loss of deep sensation, and bladder-bowel disturbances. VA dissection or occlusion is considered to play an important role in the development of PSA syndrome in the cervical cord [6], and VA dissection could lead to the sudden onset of occipital or neck pain, which is important knowledge for emergency physicians because it might be misdiagnosed as other critical diseases such as subarachnoid hemorrhage.

A characteristic of this patient is that he presented with PSA syndrome with the worst headache of his life despite not presenting with VA dissection. The pathomechanism of pain due to an intramedullary lesion has not been elucidated; however, up-regulation of neuronal activity with an increase of excitatory glutaminergic activity or a change in voltage-sensitive $\mathrm{Na}+$ channels has been suggested [11]. Furthermore, a pathological lesion in the root entry zone, which is an initial pain-processing center in the central nervous system [12], might have some associations with deafferentation pain by blockade of an afferent pathway.

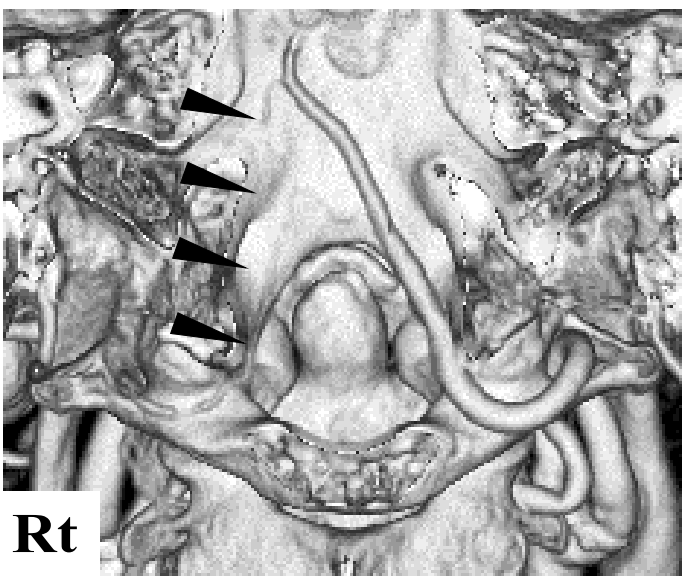

Figure 2 3D-CT angiography shows severe stenosis in the right vertebral artery (black arrowheads).

Another characteristic of this patient is that $\mathrm{ON}$ followed a severe occipital headache. ON is defined as a unilateral or bilateral paroxysmal, shooting or stabbing pain in the posterior part of the scalp, involving the distributions of the greater, lesser, and/or third occipital nerves [13]. Although contracted cervical muscles and cervical spondylosis are often implicated, some neurological disorders could also cause ON [14-19]. The ON in this patient may have been caused by an intramedullary lesion, as in previous cases $[15-17,19]$. Therefore, the exclusion of an upper cervical cord lesion in patients with $\mathrm{ON}$ might be recommended.

\section{Conclusion}

In conclusion, patients with upper cervical PSA syndrome, even without VA dissection, could present with severe occipital 
headache followed by ON. We have to consider MRI to examine upper spinal cord lesions in patients with severe occipital headache or ON along with disturbance of the deep sensation or bladder-bowel function.

\section{References}

1 Pryse-Philips W (1989) Infarction of the medulla and cervical cord after fitness exercises. Stroke 20: 292-294.

2 Gutowski NJ, Murphy RP, Beale DJ (1992) Unilateral upper cervical posterior spinal artery syndrome following sneezing. J Neurol Neurosurg Psychiatry 55: 841-843.

3 Fukuda H, Kitani M (1994) Unilateral posterior spinal artery syndrome of the upper cervical cord associated with vertebral artery occlusion. Clin Neurol 34: 1171-1174.

4 Bergqvist CA, Goldberg HI, Thorarensen O, Bird SJ (1997) Posterior cervical spinal cord infarction following vertebral artery dissection. Neurology 48: 1112-1115.

5 Masson C, Colombani JM (2002) Unilateral infarct in the posterior spinal artery territory presenting as a "clumsy hand". J Neurol 249: 1327-1328.

6 Murata K, Ikeda K, Muto M, Hirayama T, Kano O, et al. (2012) A case of posterior spinal artery syndrome in the cervical cord: a review of the clinicoradiological literature. Intern Med 51: 803-807.

7 Richard S, Abdallah C, Chanson A, Foscolo S, Baillot PA, et al. (2014) Unilateral posterior cervical spinal cord infarction due to spontaneous vertebral artery dissection. J Spinal Cord Med 37: 233-236.

8 Sakurai T, Wakida K, Nishida H (2016) Cervical Posterior Spinal Artery Syndrome: A Case Report and Literature Review. J Stroke Cerebrovasc Dis 25: 1552-1556.

9 Hsu CS, Cheng CY, Lee JD, Lee M, Huang YC, et al. (2013) Clinical features and outcomes of spinal cord infarction following vertebral

\section{Conflicts of Interest}

There are no conflicts.

artery dissection: A systemic review of the literature. Neurol Res 35: 676-683.

10 Gowers WR (1886) A manual of diseases of the nervous system. J and A Churchill, London.

11 Eide PK (1998) Pathophysiological mechanisms of central neuropathic pain after spinal cord injury. Spinal Cord 36: 601-612.

12 Melzack R, Wall PD (1965) Pain mechanisms: A new theory. Science 150: 971-979.

13 The International Classification of Headache Disorders (3rd edn). IHS Classification ICHD-3.

14 Kim K, Mizunari T, Kobayashi S, Ishii S, Teramoto A (1999) Occipital neuralgia caused by the compression of the fenestrated vertebral artery: A case report. No Shinkei Geka 27: 645-650.

15 Boes CJ (2005) C2 myelitis presenting with neuralgiform occipital pain. Neurology 64: 1093-1094.

16 Hashiguchi A, Mimata C, Ichimura H, Kuratsu J (2007) Occipital neuralgia as a presenting symptom of cervicomedullary dural arteriovenous fistula. Headache 47: 1095-1097.

17 Bruti G, Mostardini C, Pierallini A, Villani V, Modini C, et al. (2007) Neurovascular headache and occipital neuralgia secondary to bleeding of bulbocervical cavernoma. Cephalalgia 27: 1074-1079.

18 White JB, Atkinson PP, Cloft HJ, Atkinson JL (2008) Vascular compression as a potential cause of occipital neuralgia: A case report. Cephalalgia 28: 78-82.

19 De Santi L, Monti L, Menci E, Bellini M, Annunziata P (2009) Clinicalradiologic heterogeneity of occipital neuralgiform pain as multiple sclerosis relapse. Headache 49: 304-307. 